\title{
Perceived self-motion elicited by postrotary head tilts in a varying gravitoinertial force background
}

\author{
PAUL DIZIO and JAMES R. LACKNER \\ Brandeis University, Waltham, Massachusetts
}

\begin{abstract}
We measured the effects of postrotary head tilts on the perceived duration and the apparent axis of illusory self-rotation experienced following counterclockwise body rotation in high $(1.8 \mathrm{G})$, normal (1 G), and low (0 G) gravitoinertial force environments. In the absence of head movements, the duration of illusory afterrotation was shorter in $0 \mathrm{G}$ and $1.8 \mathrm{G}$ than in $1 \mathrm{G}$, and it was further shortened by $40^{\circ}$ pitch-back head movements in $1 \mathrm{G}$ and $1.8 \mathrm{G}$. Clockwise illusory afterrotation about the torso's vertical $z$-axis was always experienced in trials without postrotary head tilts. In trials with head movements, half the subjects experienced no change in this pattern; however, half experienced transient rightward roll of the torso's $z$-axis, which remained the rotation axis. The duration and extent of apparent roll were greater in $0 \mathrm{G}$ and smaller in $1.8 \mathrm{G}$ than in $1 \mathrm{G}$. We provide a functional explanation for the tendency for perceived self-rotation to be determined relative to the torso and to the gravitoinertial vertical rather than solely in relation to head position and head-fixed angular velocity sensors.
\end{abstract}

Since the early observations of Purkinje (1820), postrotary aftereffects have been generally believed to depend on head orientation. Purkinje had found that if he actively spun his body with his head erect, then upon stopping he would experience movement of himself and the visual world in the same horizontal plane. If he had been turning clockwise $(\mathrm{CW})$, he experienced $\mathrm{CW}$ self-motion and counterclockwise (CCW) visual motion upon stopping. Purkinje also found that cessation of passive body rotation was followed by apparent self-rotation opposite to the per-rotary direction, but in the same plane. Guedry and Benson (1983) have recently confirmed and extended these findings on active versus passive control of body rotation.

Purkinje reported that the aftereffects of rotation not only were dependent on head position during rotation but remained coupled to head position after rotation ceased. Thus, following active body rotation, Purkinje experienced afterrotation about a vertical axis aligned with the head; but if the head was then pitched backward, the axis of rotation remained linked to the head, and he experienced apparent rotation about a tilted axis aligned with the head. Both self-motion and visual motion were perceived in relation to the head.

These early observations were important because they pointed to the role of sensors in the head in the detection and the perception of rotation. Although Purkinje's belief that the sense of rotation was related to the lag of the brain in the cranium is now archaic, his sophisticated psychophysical observations helped spur later studies that showed the importance of the semicircular canals in the

This research was supported by NASA Contract NAS9-15147. Correspondence may be addressed to Paul DiZio, Ashton Graybiel Spatial Orientation Laboratory, Brandeis University, Waltham, MA 02254-9110. detection of angular acceleration and in giving rise to sensations of afterrotation (Mach, 1875).

Purkinje's belief that aftersensations of rotation follow head position is still commonly held and has recently received additional support (Grusser, 1984). However, several empirical and theoretical considerations have convinced us that aftersensations of body rotation are partly determined relative to the torso or to an external frame of reference such as gravity, in addition to having a tendency to remain fixed relative to the skull. First, the apparent body rotation experienced following a postrotary head movement is complex and often confusing, and we have found that more experienced subjects describe more complex aftereffects. Second, following passive body rotation, illusory afterrotation (and afternystagmus) is suppressed by head movements (Benson \& Bodin, 1966), making it briefer and harder to describe. Third, the notion that head-fixed sensory encoders of angular velocity are solely responsible for determining the perceived rotation of the entire body is untenable. Several groups of investigators have found that perceptual and oculomotor responses to a given angular velocity vary as a function of gravitoinertial force level (DiZio, Lackner, \& Evanoff, 1986, 1987b; Lackner \& Graybiel, 1981; Vesterhauge, Månsson, \& Johansen, 1984). Moreover, in recent experiments that we have carried out in order to measure the effects of postrotary head movements on nystagmus in different gravitoinertial force (G) environments (DiZio \& Lackner, 1988), our subjects spontaneously reported aftersensations of body motion that did not follow head position. In addition, DiZio and Lackner (1986) have found that the axis of visually induced illusory roll of the body may still coincide with the gravitational vertical, despite deviations of the stimulus axis of as much as $45^{\circ}$ from the vertical. 
For these various reasons, we considered it important to evaluate how aftersensations of rotation are influenced by head movement and by the magnitude and direction of the $\mathrm{G}$ force acting on the body. Our approach was to use the normal force $(1 \mathrm{G})$, free fall $(0 \mathrm{G})$, and high force $(1.8 \mathrm{G})$ phases of parabolic flight as a way of generating the desired variations in force magnitude. We passively rotated blindfolded subjects, because we wanted to eliminate the contribution of vision, oculomotor control, and voluntary control of body movement as variables.

\section{METHOD}

\section{Subjects}

Eight individuals participated; they were without known vestibular or sensory-motor abnormalities. Each had passed an FAA Class II physical and taken a physiological training course required for participation in parabolic flight experiments. Both experimenters also participated as subjects. Prior parabolic flight experience ranged from 40 ( 3 subjects) to over 12,000 parabolas.

\section{Flight Profile}

The experiment was conducted on board NASA's Boeing KC-135 aircraft during straight-and-level flight and parabolic maneuvers. Figure 1 illustrates the flight path during parabolic maneuvers. Periods of constant $0 \mathrm{G}$ and $1.8 \mathrm{G}$ alternated, each lasting approximately 20-25 sec. The parabolas were flown in five sets of eight, with a 5 -min period of straight-and-level flight between sets. During level flight, and during the constant low and high force periods, the gravitoinertial force vector was perpendicular to the deck of the aircraft.

\section{Procedure}

We generated aftereffects of rotation by exposing blindfolded subjects to sudden stops following prolonged rotation about their vertical $z$-axis; after half the sudden stops, the subjects were required to move their heads to a new position. The subject was seated in a servo-controlled rotating chair and restrained in position with a shoulder harness and lap belt. A lightweight head holder confined the subject's head movements to the pitch plane and measured their angular extent. Head movements were mechanically confined between $20^{\circ}$ ventriflexed and $20^{\circ}$ dorsiflexed, relative to a natural upright position established in $1 \mathrm{G}$. The subjects received practice in moving their heads between these end points within $0.5 \mathrm{sec}$. Prior to the start of a trial, each subject placed his head in the $20^{\circ}$ ventriflexed position. Then the chair was accelerated to $60 \% / \mathrm{sec} \mathrm{CCW}$ (leftward) and maintained at that velocity for at least $60 \mathrm{sec}$. The chair was then stopped suddenly (within . $5 \mathrm{sec}$ ), and if a head movement was required, the subject was told to make it. A few seconds before the rapid deceleration was to occur, the blindfolded subject was reminded that his tasks would be (1) either to keep his head still, or, if instructed, to move it back, (2) to describe the axis of apparent body rotation following the sudden stop and (if required) following the head movement, (3) to press a hand-held button when the sense of afterrotation ceased, and (4) to report whether an inversion illusion was experienced in $0-G$ trials.

Each in-flight test session involved six trials, two conducted in $0 \mathrm{G}$, two in $1.8 \mathrm{G}$, and two in $1 \mathrm{G}$ during straight-and-level flight. Multiple in-flight test sessions were conducted with each test subject until he had completed at least four trials for each of the $G$ levels, for stops both with and without head movements. The order of stops was balanced across the $G$ levels and the head-movement conditions. The $0-\mathrm{G}$ stops were made when a linear accelerometer indicated a stable $0-\mathrm{G}$ level, the $1.8-\mathrm{G}$ stops when the accelerometer reached $1.6 \mathrm{G}$ in the pull-up phases of the parabolas. Ground-

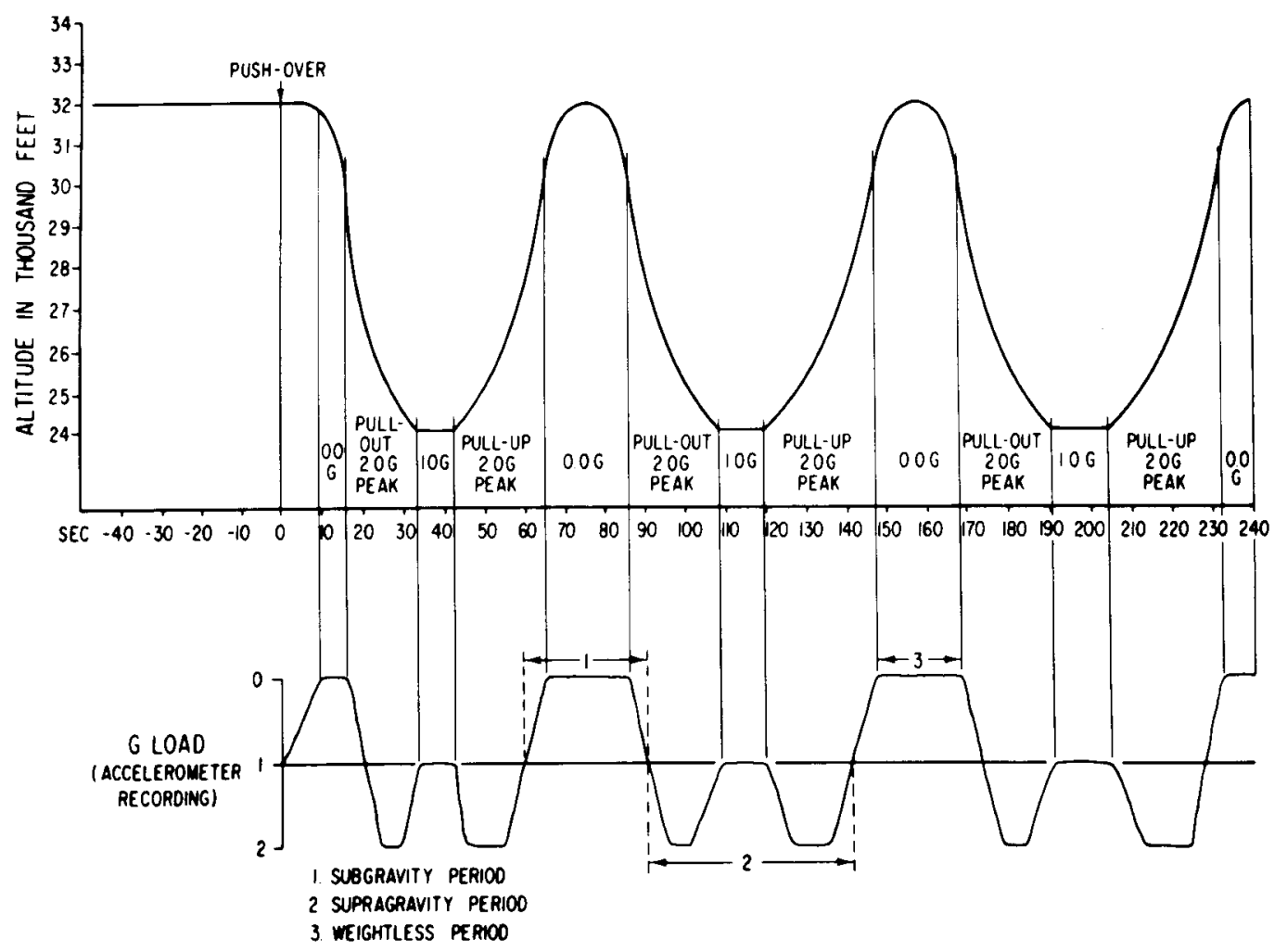

Figure 1. Schematic of the flight path of the Boeing KC-135 aircraft and of the variations in gravitoinertial force (G) magnitude generated. 
based 1-G tests were conducted prior to in-flight sessions, at least 20 days afterward.

An instrumentation tape recorder stored signals representing chair velocity, head position, gravitoinertial force level, and the subjects' verbal comments. The duration of illusory afterrotation was determined by measuring, from the tape, the time from when the chair stopped until the button was pressed. Trials were rejected from analysis if (1) the constant velocity period before the stop was less than $60 \mathrm{sec}$ in duration, (2) a head movement began more than $1 \mathrm{sec}$ after the stop, (3) the constant force period after the stop lasted less than $20 \mathrm{sec}$, or (4) the subject experienced "wobble" prior to the stop, an artifact caused by pitch or roll of the aircraft.

\section{RESULTS}

Every subject experienced aftersensations of rotation following each sudden stop, regardless of $G$ level and of whether or not a head movement was made. There were no differences between the pre- and postflight tests, so the data were lumped together as a $1-G$ ground condition. The direction of illusory afterrotation was always clockwise (rightward), opposite the per-rotary direction. However, both the duration of experienced afterrotation and the precise pattern of afterrotation were influenced by head movements and $G$ level.

The duration of illusory afterrotation was affected by gravitoinertial force level $[F(3,21)=5.21, p=.009]$, head movement $[F(1,7)=7.68, p=.047]$, and an interaction of the two $[F(3,21)=3.59, p=.002]$. Scheffé contrasts (95\% confidence) were used to compare individual conditions. In trials without head movements, the duration of illusory afterrotation was significantly shorter in $0 \mathrm{G}$ than in 1 or $1.8 \mathrm{G}$, and significantly shorter in $1.8 \mathrm{G}$ than in $1 \mathrm{G}$. In trials with head movements, the duration of illusory afterrotation was not significantly different between any of the three $G$ levels. Comparing trials with and without head movements at the same force levels, no difference was found for $0 \mathrm{G}$, but head movements significantly lowered the duration of illusory afterrotation in 1 and $1.8 \mathrm{G}$. The results are summarized in Table 1.

For all trials without postrotary head movements, regardless of $\mathbf{G}$ level, every subject experienced afterrotation about his torso's $z$-axis and perceived that axis to be vertical (see Figure 2a). As long as afterrotation

Table 1

Duration of Illusory Afterrotation Elicited Following Sudden Stops with and without Head Movement in Four Gravitoinertial Force Environments

\begin{tabular}{lccccc}
\hline & \multicolumn{4}{c}{ Condition } \\
\cline { 2 - 3 } & \multicolumn{3}{c}{ Sudden Stop } & & \multicolumn{2}{c}{$\begin{array}{c}\text { Sudden Stop and } \\
\text { Head Movement }\end{array}$} \\
\cline { 2 - 3 } \cline { 5 - 6 } & $M$ & $S E$ & & $M$ & $S E$ \\
\hline 0 G & 10.9 & 2.41 & & 10.1 & 2.26 \\
1 G (ground) & 20.7 & 4.78 & & 15.8 & 2.22 \\
1 G (in-flight) & 15.9 & 3.54 & & 11.5 & 2.32 \\
1.8 G & 14.0 & 2.56 & & 11.8 & 2.06 \\
\hline
\end{tabular}

Note $-N=8$.
A

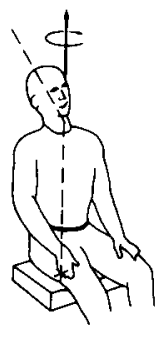

B

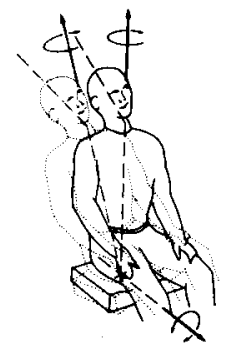

C

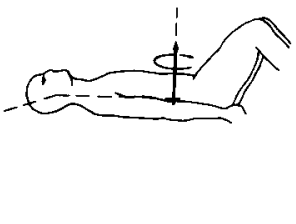

Figure 2. A. Apparent body rotation about the torso's vertical $z$-axis. B. Apparent body rotation about the torso's $z$-axis, with apparent rightward roll of the $z$-axis (dotted figure) followed by continued apparent body rotation about the vertical $z$-axis (solid figure). C. Apparent body rotation about the torso's vertical $x$-axis. Not illustrated is the fact that in $0 \mathrm{G}$, subjects sometimes felt as if they were upside-down.

lasted, its axis was perceived to be stable and vertical relative to external space, even in $0 \mathrm{G}$, where there was no mechanical or vestibular information about the vertical and some subjects felt as if they were upside-down. ${ }^{1}$ By contrast, in trials with head movements, the experience was different at each gravitoinertial force level. In 1-G trials, every subject experienced rotation about the torso's $z$-axis after head movements. Four of the subjects felt that the apparent rotation axis was always vertical. The remaining 4 experienced transient rightward roll both of the rotation axis and of themselves, commencing with the head movement and lasting for 2-3 sec; then, apparent self-rotation about the torso's $z$-axis continued to be experienced, but that axis was again perceived to be vertical (see Figure 2b). In 1.8-G trials, each subject reported experiences similar to those in $1 \mathrm{G}$. The same four subjects reported that the axis of rotation always seemed vertical, and the 4 who had experienced transient rightward body roll in $1 \mathrm{G}$ reported its duration and extent to be less in $1.8 \mathrm{G}$ than in $1 \mathrm{G}$. In $0 \mathrm{G}$, the subjects also reported experiences after the head movements that were similar to those in $1 \mathrm{G}$, except that the 4 individuals who felt rightward body roll reported its duration and extent to be greater than in $1 \mathrm{G} ; 1$ of the 4 experienced body roll but not roll of the apparent rotation axis, so that he felt he was on his back rotating about his torso's $x$-axis (see Figure 2c). After each of their reports, the subjects were asked whether they had experienced the axis of apparent body rotation as moving with and coinciding with their head movements; all subjects denied having had such an experience. There was no difference in prior parabolic flight experience between the subjects who always experienced vertical axis rotation following head movements and those who experienced transient rightward roll.

Overall, in all G levels, with and without head movements, the apparent axis of body afterrotation was perceived as vertical except for a 2-3 sec period following head movements for some subjects. Even in this 2-3 sec period, the axis of apparent rotation did not follow head 
tilt. The axis of apparent self-rotation coincided with the $z$-axis of the trunk for all subjects when they were tested in 1 and $1.8 \mathrm{G}$ and for all but one in $0 \mathrm{G}$.

\section{DISCUSSION}

The present findings indicate that aftersensations of body rotation are not always localized with respect to the head and are influenced by the $G$ level. In fact, none of our subjects, regardless of the $G$ level, experienced their axis of apparent self-rotation as following head position when they moved their heads. Accordingly, the present observations do not support the classic notion that the semicircular canals alone determine the apparent orientation of the axis of self-rotation relative to the head and the apparent velocity of rotation. These observations do provide, however, some insight into the way in which canalicular, otolithic, somatosensory, and motor information about head and body orientation are integrated.

In each trial, regardless of whether voluntary pitch head movements were made, the horizontal semicircular canals signaled clockwise rotation following the sudden stop. This input was the sensory source of the clockwise sensation of afterrotation that all of the subjects experienced. In trials not involving head movements, the duration of sensed afterrotation was least in $0 \mathrm{G}$, intermediate in $1.8 \mathrm{G}$, and longest in $1 \mathrm{G}$. In other experiments, the time constants of slow phase velocity decay of the nystagmoid eye movements elicited by sudden stops showed precisely the same pattern for the same test conditions, while the peak velocity of nystagmus was constant (DiZio et al., 1987b). Taken with the present observations, these findings indicate that in non-1-G force environments, at least initially, the central expression of semicircular canal activity is modulated in terms of its effects on apparent body rotation and oculomotor control. The nature of this modulation is consistent with a decrease in the closed-loop time constant of the velocity storage integrator (Raphan, Matsuo, \& Cohen, 1979).

In trials with postrotary head movements, three facts require explanation. The first involves the shorter sensations of afterrotation in 1 and $1.8 \mathrm{G}$ but not in $0 \mathrm{G}$; the second is the transient, rightward roll of the rotation axis consistently experienced by half the subjects; the third is the strong tendency to perceive a constant orientation of the axis of apparent rotation relative to the torso and external space, even in $\mathbf{0}$ G. The first two issues can be explained in terms of events triggered by a discordance between semicircular canal and graviceptor signals, but the third issue requires a different type of explanation.

With regard to the first issue, note that, after the sudden stops, the horizontal canal signals were discordant with the otolith signals in 1 and $1.8 \mathrm{G}$; the former were signaling "clockwise, $z$-axis rotation" and the latter were signaling "steady state, head tilted from the vertical." If the body had actually been rotating about the head on an off-vertical axis, then the signals from the otolith organs and somatosensory mechanoreceptors would have been continuously changing because of the continual reorientation of the body in relation to the gravitoinertial force vector. The absence of such a changing linear acceleration input after postrotary head tilts indicates that the individual is not rotating about the $z$-axis of his tilted head. The presence of a discordance between canal and graviceptor signals after postrotary head tilts has been known for some time to lead to shorter sensations of selfmotion (Benson \& Bodin, 1966). In 1.8 G, if off-vertical rotation were really occurring, the graviceptor signals would also be continuously changing but with greater amplitude; consequently, there is a greater discordance than in $1 \mathrm{G}$, because the graviceptor signals are static and greater in intensity, leading to sensations of self-motion's being shorter in $1.8 \mathrm{G}$ than in $1 \mathrm{G}$. In $0 \mathrm{G}$, a changing otolith signal is not possible after repositioning of the head, so its absence does not alter the duration of the experienced afterrotation. In both 1 and $1.8 \mathrm{G}$, the changes in duration of illusory afterrotation elicited by head tilts are consistent with a decrease in the time constant of the velocity storage integrator.

The duration of transient rightward roll of the $z$-axis of the trunk experienced following head movements is probably also related to a canal-graviceptor discordance. When the head is dorsiflexed, cervical, proprioceptive, motor, vertical semicircular canal, and (in 1 and $1.8 \mathrm{G}$ ) otolith signals indicate the change in head position. The persisting horizontal canal activity in conjunction with signals indicating the reoriented, dorsiflexed position of the head on the torso would specify body rotation with components of rightward roll and rightward yaw vis à vis the torso and gravity. The absence of confirming otolithic and somatosensory cues indicates, as explained above, that the body cannot actually be rolling rightward, and it leads to a rapid attenuation of the roll component of the experience while the experienced yaw continues for about $10 \mathrm{sec}$. The pattern of semicircular canal stimulation that our subjects encountered when making postrotary head tilts is similar, in terms of generating a roll component of apparent motion, to what would be encountered during per-rotary head tilts, which generate Coriolis, crosscoupling stimulation (Guedry \& Montague, 1961; Schubert, 1954). Consistent with the present results, we have found that the severity of apparent disorientation elicited by Coriolis, cross-coupling stimulation is a function of G level (DiZio, Lackner, \& Evanoff, 1987a; Graybiel, Miller, \& Homick, 1977).

A different explanation is needed for the third issue raised above-the tendency to maintain a fixed apparent rotation axis vis à vis the torso after postrotary head tilts. Note that when proprioceptors indicate a change in the angle between head and torso, it is usually because of a change in head orientation rather than a change in torso orientation relative to space. Thus, it would be functionally useful to cancel changes in the apparent rotation axis of the entire body that would result from voluntary head tilts, so as to stabilize apparent torso motion relative to space. The mechanism we propose is analogous to that 
of von Holst and Mittelstaedt (1950) concerning how extraretinal information about eye movements can be used to stabilize apparent visual direction relative to the head. Our proposal has considerable functional utility, because locomotion as well as the control of arm movements is primarily dependent on torso rather than head orientation relative to external space; and the center of mass of the body that has to be controlled is in the torso. Monitoring body position vis à vis the torso and gravity thus allows orientational specification of both.

The differences between our findings and those of earlier workers concerning the consequences of postrotary head movements probably arise because our subjects were blindfolded. In fact, experiments we are pursuing suggest, in accord with the reports of Purkinje and Grusser, that the apparent motion of the visual array after body rotation is dependent on head position. Such a dependency might be expected, because the semicircular canals are fixed with respect to the head, and fibers from the various canals innervate oculomotor neurons whose activity leads to specific patterns of eye movements in relation to the skull; consequently, eye movements and visual input associated with canal stimulation, as well as oculomotor commands necessary to suppress a vestibular nystagmus (cf. Evanoff \& Lackner, 1987), might be expected to have a strong link to head position. It should be noted, however, that even nystagmus is subject to a tendency to be determined in reference to the gravitoinertial vertical. Raphan and Cohen (1987) have shown that velocity storage integrator activity is enhanced about the axis corresponding to the gravitational vertical. We are exploring this linkage by measuring whether there is a change in the plane of compensatory postrotary nystagmus vis à vis the head when head orientation is manipulated.

\section{REFERENCES}

Benson, A. J., \& Bodin, M. A. (1966). Comparison of the effects of the direction of the gravitoinertial acceleration on post-rotational responses in yaw, pitch and roll. Aerospace Medicine, 37, 889-897.

DiZio, P., \&Ackner, J. R. (1986). Perceived orientation, motion, and configuration of the body during viewing of an off-vertical, rotating surface. Perception \& Psychophysics, 39, 39-46.

DiZıo, P., LACKNER, J. R. (1988). The effects of gravitoinertial force level and head movements on post-rotational nystagmus and illusory after-rotation. Experimental Brain Research, 70, 485-495.

DiZio, P., LACkner, J. R., \& Evanoff, J. N. (1986, February). The influence of gravitoinertial force level on oculomotor and perceptual responses to sudden stop stimulation. Paper presented at the 7th International Man in Space Symposium: Physiologic Adaptation of Man in Space. Houston, Texas.

DiZio, P. A., Lackiner, J. R., \& Evanoff, J. N. (1987a). The influence of gravitoinertial force level on oculomotor and perceptual responses to Coriolis, cross-coupling stimulation. Aviation, Space \& Environmental Medicine, 58(9, Suppl. A), 218-223.

DiZıo, P. A., Lackner, J. R., \& Evanoff, J. N. (1987b). The influence of gravitoinertial force level on oculomotor and perceptual responses to sudden stop stimulation. Aviation, Space \& Environmental Medicine, 58(9, Suppl. A), 224-230.

EVANOFF, J. N., \& LACKNER, J. R. (1987). Influence of maintained ocular deviation on the spatial displacement component of the oculogyral illusion. Perception \& Psychophysics, 42, 25-28.

Graybiel, A., \& KellogG, R. S. (1967). The inversion illusion in parabolic flight: Its probable dependence on otolith function. Aerospace Medicine, 38, 1099-1103.

Graybiel, A., Miller, E. F., \& Homick, J. L. (1977). Experiment M-131: Human vestibular function. In R. S. Johnston \& L. F. Dietlein (Eds.), Biomedical results from Skylab: Section II (NASA Publication No. SP-377, pp. 74-103). Washington, DC: U.S. Government Printing Office.

Grusser, O.-J. (1984). J. E. Purkyne's contributions to the physiology of the visual, the vestibular and the oculomotor systems. Human Neurobiology, 3, 129-144.

GUEDRY, F. E., \& BENSON, A. J. (1983). Modification of per- and postrotational responses by voluntary motor activity of the limbs. Experimental Brain Research, 52, 190-198.

Guedry, F. E., \& Montague, E. K. (1961). Quantitative evaluation of the vestibular Coriolis reaction. Aerospace Medicine, 32, 487-500.

LACKNER, J. R. (in press). Perceived orientation in the weightless phase of parabolic flight maneuvers. Perception.

LACKNER, J. R., \& GRA YBIEL, A. (1981). Variations in gravitoinertial force level affect the gain of the vestibulo-ocular reflex: Implications for the etiology of space motion sickness. Aviation, Space \& Environmental Medicine, 52, 154-158.

LACKNer, J. R., \& Graybiel, A. (1983). Perceived orientation in free fall depends on visual, postural, and architectural factors. Aviation, Space \& Environmental Medicine, 54, 47-51.

МАCH, E. (1875). Grundlinien der Lehre von den Bewegungsempfindunger. Leipzig: Wilhelm Engelman.

PURKINJE, J. (1820). Beitrage zur naheren Kenntnis der Schwindels aus heautognostischen Daten. Medizinische Jahrbulcher des K.K. Österreichischen Staates, 6, 79-125.

Raphan, T., Matsuo, V., Cohen, B. (1979). Velocity storage in the vestibulo-ocular reflex arc (VOR). Experimental Brain Research, $35,229-248$.

RAPHAN, T., \& CohEN, B. (1987). Effects of gravity on the principle axes of velocity storage in three dimensions. Society for Neuroscience Abstracts, 13, 1225.

SCHUBERT, G. (1954). Coriolis nystagmus. Joumal of Aviation Medicine, 25, 257-259.

Vesterhauge, S., MÁnsson, A., * Johansen, T. S. (1984). Vestibular and oculomotor function during $G$, variations (AGARD CP 372, Motion sickness: Mechanisms, prediction, prevention and treatment, 24.124.4). NATO Advisory Group for Aerospace Research and Development, Conference Proceedings.

von Holst, E., \& MitTelstaedT, H. (1950). Das Reafferenzprinzip. Wechselwirkungen zwischen Zentralnervensystem und Peripherie. Naturwissenschaften, 37, 464-476.

\section{NOTE}

1. When the force level changed from 1.8 to $0 \mathrm{G}$, the blindfolded subjects felt an inversion of their orientation. The feeling of being upsidedown continued throughout illusory afterrotation elicited by a sudden stop and lasted for 20 to $25 \mathrm{sec}$, until the next force transition. This is consistent with descriptions of the inversion illusion in parabolic flight (Graybiel \& Kellogg, 1967; Lackner, 1988; Lackner \& Graybiel, 1983). Three subjects experienced inversion illusions during $22 \%$ of all 0-G trials.

(Manuscript received June 17, 1988; revision accepted for publication January $17,1989$. 